\title{
Comparação de dois ésteres de estradiol como indutores da ovulação sobre o diâmetro folicular e a taxa de gestação de bovinos leiteiros submetidos a programa de Inseminação Artificial em Tempo Fixo
}

\author{
Comparison of two esters estradiol as inductors on ovulation follicular diameter and \\ pregnancy rate of dairy cattle subject to program of FTAI
}

\author{
FRANÇA, Lidomar Marton ${ }^{1}$; RODRIGUES, Alexandra Soares ${ }^{2} *$; BRANDÃO, Luiz \\ Gustavo Neves ${ }^{1}$; LOIOLA, Marcus Vinícius Galvão ${ }^{3}$; CHALHOUB, Marcos ${ }^{3}$; \\ FERRAZ, Priscila Assis ${ }^{3}$; BITTENCOURT, Rodrigo Freitas ${ }^{3}$; JESUS, Edivânia \\ Oliveira de ${ }^{3}$; RIBEIRO FILHO, Antonio de Lisboa ${ }^{3}$
}

\footnotetext{
${ }^{1}$ Faculdade de Tecnologia e Ciências, Feira de Santana, Bahia, Brasil.

${ }^{2}$ Universidade Federal do Oeste da Bahia, Centro Multidisciplinar Campus Barra, Barra, Bahia, Brasil.

${ }^{3}$ Universidade Federal da Bahia, Escola de Medicina Veterinária e Zootecnia, Departamento de Anatomia, Patologia e Clínicas, Salvador, Bahia, Brasil.

*Endereço para correspondência: alexandra.rodrigues@ufob.edu.br
}

\section{RESUMO}

Objetivou-se com este trabalho avaliar o efeito da utilização de dois ésteres de estradiol, sobre o Diâmetro do Folículo Ovulatório (DFOL) e taxa de gestação de fêmeas bovinas leiteiras submetidas a um programa de Inseminação Artificial em Tempo Fixo (IATF). Para tanto, 94 fêmeas mestiças Holandês-Gir foram submetidas ao seguinte protocolo hormonal, iniciado em um dia aleatório, D0, por meio da inserção de um dispositivo intravaginal de progesterona $(\mathrm{P} 4)$ associado à $2 \mathrm{mg}$ de Benzoato de Estradiol (BE) via intramuscular (i.m.). No $\mathrm{D} 8$, os dispositivos foram removidos e administrados $500 \mu \mathrm{g}$ de Cloprostenol sódico prostaglandina - F2 $\alpha$ e 300 UI de Gonadotrofina Coriônica equina (eCG) i.m. Neste momento, os animais foram divididos em dois grupos experimentais: 50 vacas foram alocadas no grupo Cipionato de Estradiol (CE) e receberam $1 \mathrm{mg}$ dessa substância via i.m., enquanto outras 44 receberam $1 \mathrm{mg}$ de BE i.m., 24 horas após a retirada dos dispositivos (D9), sendo chamadas grupo BE. No D10, foram realizadas as inseminações e, previamente a cada procedimento de IA, foi executada a mensuração do DFOL por meio de ultrassonografia transretal. O diagnóstico de gestação foi realizado 55 dias após as IATFs. Não houve diferença significativa entre as médias de DFOL encontradas para os grupos
BE e CE sendo, respectivamente, $11,45 \pm 2,34$ e $10,71 \pm 2,43 \mathrm{~mm}$. $\mathrm{O}$ grupo $\mathrm{BE}$ também apresentou um índice de gestação de 34,10\%, semelhante ao grupo CE que foi de $40,00 \%$. Conclui-se que o CE pode substituir o $\mathrm{BE}$ em protocolo de IATF em vacas leiteiras, representando uma alternativa para simplificar o protocolo de IATF.

Palavras-chave: benzoato de estradiol, cipionato de estradiol, fertilidade

\section{SUMMARY}

This study aimed to evaluate the effect of using two estradiol esters, on the diameter of the ovulatory follicle (DFOL), and pregnancy rates of dairy cows submitted to FTAI program. Therefore, 94 females crossbred Dutch-Gir were subjected to the following hormone protocol started on a random day D0, by insertion of an intravaginal device of progesterone (P4) associated to $2.0 \mathrm{mg}$ of estradiol benzoate (EB) intramuscular (im). In D8, the devices were removed and $500 \mu \mathrm{g}$ of sodium cloprostenol prostaglandin $-\mathrm{F} 2 \alpha$ was administered and 300 IU of equine chorionic gonadotropin (eCG) i.m. At this time the animals were divided in two groups: 50 cows were allocated to EC group and 
received $1 \mathrm{mg}$ of estradiol cypionate i.m., while the other 44 received $1.0 \mathrm{mg}$ of EB i.m. 24 hours after removal of the devices (D9), being called the BE Group. At D10, inseminations were carried out, and prior to each IA procedure were performed trans rectal ultrasonography to measure the DFOL. Pregnancy diagnosis was performed 55 days after the FTAI. There was no significant difference between measurement of DFOL found for the $\mathrm{BE}$ and $\mathrm{CE}$ groups being, respectively, $11.45 \pm 2.34$ and $10.71 \pm 2,43 \mathrm{~mm}$. The BE group also showed a $34.10 \%$ pregnancy rate, similar to the EC group which was $40.00 \%$. It is concluded that $\mathrm{CE}$ can replace the $\mathrm{BE}$ in TAI protocol in dairy cows, representing an alternative to simplify the FTAI protocol.

Keywords: estradiol benzoate, estradiol cypionate, fertility

\section{INTRODUÇÃO}

A eficiência reprodutiva é um dos fatores que mais contribui para melhorar o desempenho e a lucratividade dos rebanhos leiteiros (KOZICK et al., 2005).

A inserção de biotecnologias da reprodução como a Inseminação Artificial (IA) figura como importante ferramenta para melhorar a eficiência reprodutiva do rebanho. Entretanto, as falhas na detecção de estro e o anestro pós-parto constituem os principais gargalos para o sucesso dos programas de IA convencional (INFORZATTO et al., 2008). Para tanto, o emprego de fármacos permite sincronizar o estro e a ovulação proporcionando a Inseminação Artificial em tempo fixo (IATF) (BARUSELLI, 2004; PINHEIRO et al., 2009).

$\mathrm{O}$ uso da IATF possibilita à fazenda uma produção programada, concentração do período de parto, além da diminuição do intervalo entre partos, proporcionando maior rentabilidade para a propriedade (INFORZATTO et al., 2008).
Atualmente, existem diversos protocolos de IATF com variações de hormônios, sendo que os mais utilizados são aqueles à base de estrógenos e progestágenos para sincronizar a onda folicular (BÓ et al., 1995), seguida do uso da prostaglandina no momento da remoção do progestágeno para permitir o término da fase luteínica de forma sincronizada e, posteriormente, faz-se a indução da ovulação utilizando $\mathrm{GnRH}$ ou análogos e os estrógenos e seus ésteres (BÓ et al., 2003; PINHEIRO et al., 2009). Dentre os fármacos utilizados para a indução da ovulação, o éster de estradiol, por ser eficiente e economicamente viável, possui amplo emprego em programas comerciais de IATF (RODRIGUES, 2011).

Diante deste quadro, alguns pesquisadores demonstraram que em vacas de corte o Benzoato de Estradiol foi eficiente em induzir a ovulação quando aplicado $24 \mathrm{~h}$ após a remoção da progesterona (BARUSELLI et al., 2006), no entanto, outros experimentos demonstraram que a substituição deste, pelo Cipionato de Estradiol, aplicado no momento de retirada da progesterona (AYRES et al., 2006), eliminaria o manejo adicional, tornando a execução dos protocolos mais simples (CREPALDI, 2009).

As taxas de sucesso com a IATF, em termos de gestação, encontram-se estacionadas em torno de 35\% para bovinos de leite. Neste contexto, alguns ajustes nos protocolos podem proporcionar uma melhora nos resultados (LUCY, 2011).

Portanto, objetivou-se avaliar o efeito da utilização de dois ésteres de estradiol sobre o Diâmetro do Folículo Ovulatório (DFOL) e a taxa de gestação de fêmeas bovinas leiteiras submetidas a um programa de IATF. 


\section{MATERIAIS E MÉTODOS}

O experimento foi realizado entre os meses de agosto e outubro de 2013 na fazenda Gameleira, localizada no município de Riachão do Jacuípe/BA, propriedade situada no semiárido baiano com latitude de $11^{\circ} 48^{\prime} 25^{\prime \prime}$ sul e longitude de $39^{\circ} 23^{\prime} 08^{\prime \prime}$ oeste. A propriedade adota o sistema semi-intensivo de criação em pastagem de capim buffel e urochloa, suplementação mineral e água ad libitum. Foram utilizadas 94 fêmeas multíparas lactantes mestiças de Holandês x Gir com grau sanguíneo $1 / 4,1 / 2,3 / 8,3 / 4$ e $5 / 8$, com média de idade de 6,69 $\pm 3,60$ anos, Escore de Condição Corporal (ECC), atribuído em uma escala (1-5), de 3,38 \pm 0,51 e intervalo pós-parto de 81,36 \pm 32,04 dias. Previamente ao início do experimento, todos os animais foram submetidos a exame clínico ginecológico e ultrassonografia transretal utilizando transdutor linear de 5,0MHz (Mindray, DP2200vet, São Paulo, Brasil), sendo utilizado apenas animais com ausência de qualquer anormalidade do trato reprodutivo.

Para a realização deste experimento, os animais foram distribuídos uniformemente em relação ao ECC, intervalo pós-parto e graus de sangue em dois grupos, sendo submetidos ao seguinte protocolo hormonal: no dia 0 , as fêmeas receberam um dispositivo intravaginal de progesterona-P4 (DIB $\AA$, Zoetis, São Paulo, Brasil) associado a $2 \mathrm{mg}$ de Benzoato de Estradiol (Gonadiol ${ }^{\circledR}$, Zoetis, São Paulo, Brasil) via intramuscular (i.m.). No D8, os dispositivos foram removidos e administrados $500 \mu \mathrm{g}$ de Cloprostenol sódico - prostaglandina - F2 $\alpha$ (Ciosin $\AA$, MSD saúde Animal, São Paulo, Brasil) i.m. e 300 UI de gonadotrofina coriônica equina-eCG (Novormon $\AA$, Zoetis, São Paulo, Brasil) i.m. Neste momento, os animais foram divididos em dois grupos experimentais: 50 vacas foram alocadas no grupo $\mathrm{CE}$ e receberam $1 \mathrm{mg}$ de Cipionato de Estradiol (ECP®), Zoetis, São Paulo, Brasil) i.m.; enquanto outras 44 fêmeas foram classificadas como grupo $\mathrm{BE}$ sendo tratadas com $1 \mathrm{mg}$ de $\mathrm{BE}$ i.m., 24 horas após a remoção do dispositivo intravaginal (D9). No D10, 48 a 56 horas após a remoção do dispositivo de progesterona, foram realizadas as inseminações e, previamente a cada procedimento de IA, foi executada a mensuração do DFOL por meio de ultrassonografia transretal, utilizando-se um transdutor linear com frequência de $5,0 \mathrm{MHz}$ (Mindray, DP2200vet, São Paulo, Brasil) (Figura 1).

Todas as inseminações foram realizadas por um mesmo técnico utilizando doses de sêmen criopreservados de um mesmo touro da raça Girolanda. No momento da inseminação, o sêmen foi descongelado a $37^{\circ} \mathrm{C}$ por 30 segundos.

O diagnóstico de gestação foi realizado por ultrassonografia transretal 55 dias após a IATF, utilizando-se um transdutor linear com frequência de $5,0 \mathrm{MHz}$ (Mindray, DP2200vet, São Paulo, Brasil). Foi considerado diagnóstico de gestação positivo a presença de um feto viável e com batimento cardíaco detectado.

A taxa de gestação foi calculada pela proporção de vacas gestantes sobre o total de vacas inseminadas. Para a análise estatística dos dados, foi utilizado o Statistical Package for Social Science (SPSS, versão 19), considerando nível de significância de 5\%.

Para avaliar a diferença entre as médias do Diâmetro do Folículo Ovulatório (DFOL) nos grupos $\mathrm{BE}$ e $\mathrm{CE}$, foi utilizada a análise de variância por meio do teste $\mathrm{T}$ de Student. Já a taxa de gestação foi comparada entre os grupos utilizando-se uma análise de dispersão de frequências por meio do teste do Quiquadrado $\left(\chi^{2}\right)$. 


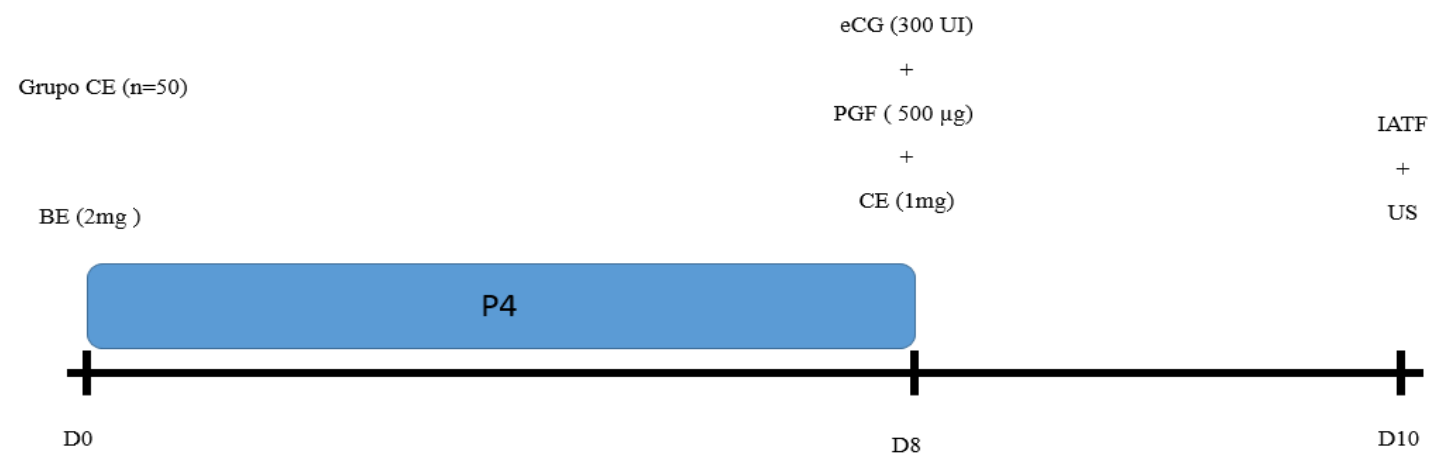

Grupo BE $(\mathrm{n}=44)$

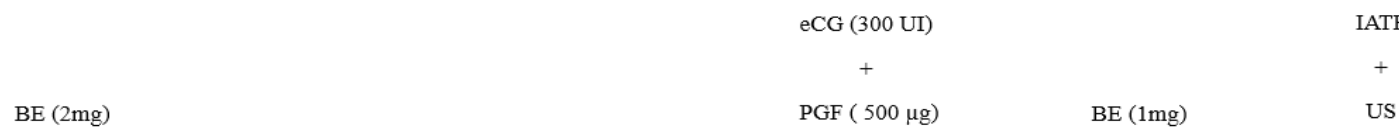

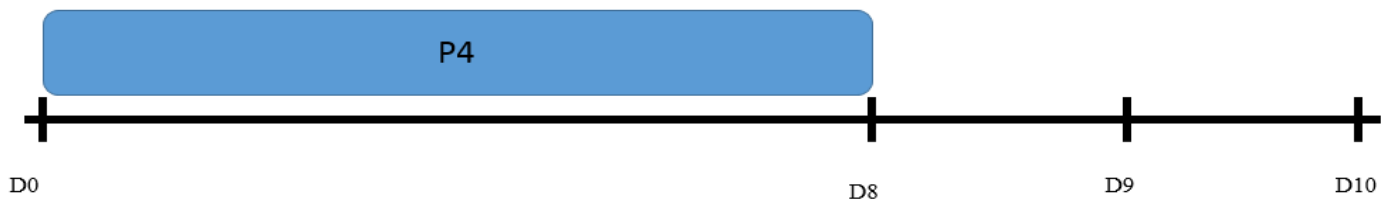

$\mathrm{BE}=$ Benzoato de Estradiol; $\mathrm{CE}=$ Cipionato de Estradiol; $\mathrm{eCG}=$ Gonadotrofina Coriônica equina; $\mathrm{PGF}=$ Prostaglandina; IATF = Inseminação Artificial em Tempo Fixo; US = Ultrassonografia.

Figura 1. Representação esquemática dos protocolos de sincronização utilizado para realização da Inseminação Artificial em Tempo Fixo (IATF) em fêmeas bovinas mestiças Holandês-Gir

\section{RESULTADOS E DISCUSSÃO}

A média geral do DFOL foi $10,99 \pm$ 2,41mm. Na comparação do DFOL entre os tratamentos, não houve diferença significativa e as médias encontradas para os grupos BE e CE foram, respectivamente, $11,45 \pm 2,34 \mathrm{e}$ $10,71 \pm 2,43 \mathrm{~mm}$ (Tabela 1$)$.

Resultados similares foram descritos por Andrade et al. (2012) em um estudo com IATF utilizando distintos ésteres de estradiol para indução da ovulação. Estes autores verificaram que o DFOL não diferiu entre os tratamentos que empregou CE simultânea a remoção da progesterona ou BE 24h após, sendo, respectivamente, de $13,03 \pm 2,24 \mathrm{~mm}$ e $12,40 \pm 1,34 \mathrm{~mm}$, porém estes pesquisadores trabalharam com fềmeas Nelore.

Da mesma forma, Sales et al. (2012), em experimentos semelhantes em vacas Nelore, não encontraram diferença entre o DFOL de fêmeas tratadas com CE no momento da remoção do dispositivo de progesterona que foi de $13,90 \pm 0,40$ ou BE 24h após que foi de $13,10 \pm 0,40 \mathrm{~mm}$.

Estes resultados se assemelham aqueles reportados por Freitas et al. (2013) que, trabalhando com vacas lactantes da raça Holandesa, avaliou a eficiência da utilização do $\mathrm{BE}$ ou $\mathrm{CE}$ como indutores de ovulação em protocolo de sincronização da ovulação para IATF. Estes autores observaram que não houve diferença em relação à média de $\mathrm{DFOL}$ para os animais que receberam $\mathrm{CE}$ no dia oito $(15,0 \pm 0,5 \mathrm{~mm})$ e o $\mathrm{BE}(13,9 \pm 0,5 \mathrm{~mm})$ no dia nove do protocolo. Contudo, os 
Rev. Bras. Saúde Prod. Anim., Salvador, v.16, n.4, p.958-965out../dez.., 2015 http://www.rbspa.ufba.br

resultados acima esboçados demonstram uma média geral de DFOL numericamente superior à obtida no presente estudo sugerindo que esta divergência ocorreu devido a este último ter sido realizado com fêmeas mestiças.

A similaridade entre os diâmetros foliculares formados pela ação de ambos os indutores da ovulação indicam que os mesmos foram eficazes em estimular um crescimento folicular proporcionando a formação de DFOLs acima de $10,00 \mathrm{~mm}$, os quais, conforme demonstrado por Sartori et al. (2001), apresentam responsividade a ação do LH e a ovulação em fêmeas leiteiras.

Tabela 1. Média e desvio-padrão (S) do Diâmetro do Folículo Ovulatório (DFOL) nos grupos de fêmeas submetidas aos tratamentos de indução da ovulação com Cipionato de Estradiol (CE) no momento da remoção do dispositivo de progesterona (P4) ou Benzoato de Estradiol (BE) 24 horas após a remoção da P4

\begin{tabular}{lcc}
\hline Tratamento & $\mathrm{N}$ & DFOL $(\mathrm{mm})$ \\
\hline GRUPO BE & 44 & $11,45 \pm 2,34$ \\
GRUPO CE & 50 & $10,71 \pm 2,43$ \\
\hline Total & 94 & $10,99 \pm 2,41$
\end{tabular}

Médias comparadas pelo teste T de Student a 5\% de probabilidade.

No que se refere à taxa de gestação, de um total de 94 animais, 35 ficaram gestante perfazendo uma taxa de gestação geral de 37,20\%. Não foi verificada diferença significativa entre os grupos experimentais, o grupo $\mathrm{BE}$ apresentou um índice de $34,10 \%$, enquanto no grupo $\mathrm{CE}$ apresentou $40,00 \%$ de taxa de gestação (Tabela 2).

Tabela 2. Taxa de gestação por exame ultrassonográfico aos 55 dias após IATF de fêmeas mestiças Holandesas submetidas aos tratamentos de indução da ovulação com Cipionato de Estradiol (CE) no momento da remoção do dispositivo de progesterona ou Benzoato de Estradiol (BE) 24h após a remoção da $\mathrm{P} 4$

\begin{tabular}{llc}
\hline Tratamento & $\mathrm{N}$ & Taxa de Gestação \\
\hline GRUPO BE & 44 & $34,10 \%(15 / 44)$ \\
GRUPO CE & 50 & $40,00 \%(20 / 50)$ \\
\hline Total & 94 & $37,20 \%(35 / 94)$ \\
\hline
\end{tabular}

Médias comparadas pelo teste qui-quadrado $\left(\chi^{2}\right)$ a $5 \%$ de probabilidade.

Apesar das condições climáticas adversas que a região atravessou nos últimos anos, onde as pastagens se mostraram bem deficientes podendo comprometer $\mathrm{o}$ desempenho reprodutivo das fêmeas, a média de prenhez ficou dentro dos valores esperados para programas comerciais de IATF em bovinos leiteiros que é de $35 \%$. Estas condições são mais críticas em vacas de leite criadas em regiões tropicais, as quais normalmente apresentam diminuição na qualidade oocitária e queda da taxa de gestação em 
programas de IA (RODRIGUES et al., 2008).

Estes índices foram semelhantes aos observados por Pfeifer et al. (2005), os quais obtiveram em média $50 \%$ de taxa de gestação em vacas lactantes submetidas a um protocolo a base de progesterona com a utilização de CE no momento da remoção do dispositivo de progesterona ou BE $24 \mathrm{~h}$ após. Adicionalmente, foi verificada que administração de CE no momento da retirada do implante de progesterona resultou em uma excelente sincronia entre o estro e a ovulação (AYRES et al., 2006).

Seguindo a mesma linha de pesquisa, Crepaldi (2009), ao avaliar a taxa de gestação em vacas Nelore submetidas a um protocolo de sincronização utilizando CE como indutor da ovulação aplicado concomitante à remoção da progesterona ou BE $24 \mathrm{~h}$ depois, encontraram resultados equivalentes ao deste estudo com índices de gestação de $47,30 \%$ para o tratamento com CE e $53,30 \%$, para o tratamento com BE.

De forma semelhante, Sales et al. (2012) também encontraram taxas de gestação parecidas em protocolos de IATF em vacas Nelore utilizando o $\mathrm{CE}$ concomitante a remoção da progesterona ou o BE 24h após, sendo respectivamente, de $61,80 \%$ e $57,50 \%$. Estes autores justificaram tal achado, ao fato de ambos os fármacos serem eficientes na indução da ovulação e formação de corpos lúteos capazes de favorecer $o$ estabelecimento e a manutenção da gestação.

Corroborando aos resultados do presente estudo, Rodrigues et al. (2008) encontraram taxa de gestação de $41,60 \%$, quando empregaram $1 \mathrm{mg}$ de $\mathrm{CE}$ como indutor da ovulação em um protocolo de IATF à base de progesterona em vacas leiteiras. Contudo, em seu delineamento experimental, os autores não comparam os efeitos do BE como indutor. Estes autores concluíram que a indução da ovulação com CE foi eficiente em um programa de IATF em fêmeas bovinas leiteiras por reduzir o número de manejos com os animais.

Os resultados encontrados neste trabalho foram superiores aos achados por Rodrigues et al. (2011). Em um experimento realizado com vacas leiteiras holandesas de alta produção no período de verão, testaram a utilização de implante de progesterona novo com aplicação de Cipionato de Estradiol, concomitantemente com a retirada da progesterona com aplicação de eCG, realizando diagnóstico de gestação aos 30 e 60 dias, obtendo taxas de gestação de $19,2 \%$ e $17,3 \%$. Os mesmos atribuem as baixas taxas de concepção provavelmente devido ao estresse calórico pelo qual os animais passaram.

Danieli et al. (2008) trabalhando com protocolo semelhante, utilizando $\mathrm{CE}$ no momento da retirada do dispositivo de progesterona, verificaram em vacas e novilhas mestiças leiteiras taxas de gestação de $42,5 \%$ para novilhas e $50 \%$ para vacas, assemelhando-se aos resultados desta pesquisa.

No entanto, Cardoso et al. (2010), utilizando Cipionato de Estradiol no dia da retirada do implante, obtiveram taxa de $35,7 \%$ em vacas mestiças Zebu $\mathrm{x}$ Holandês no estado de São Paulo.

Contudo, a maioria dos trabalhos existentes na literatura aborda o tema em animais de corte, existindo uma deficiência destes estudos em fêmeas bovinas leiteiras. Os resultados mostraram que a utilização do $\mathrm{CE}$ no momento da remoção do dispositivo de progesterona ou $\mathrm{BE} 24$ horas após não afetaram o DFOL e as taxas de gestação do protocolo de IATF e ambos os fármacos podem ser empregados eficientemente em programas de sincronização de vacas mestiças leiteiras.

A substituição do BE por um tratamento com CE como indutor da ovulação 
Rev. Bras. Saúde Prod. Anim., Salvador, v.16, n.4, p.958-965out../dez.., 2015 http://www.rbspa.ufba.br ISSN 15199940

parece não comprometer os índices reprodutivos em protocolos de IATF à base de progesterona, representando uma alternativa para simplificar os programas de sincronização em fêmeas mestiças Holandês-Gir.

\section{REFERÊNCIAS}

ANDRADE, B.H. de A.; FERRAZ, P.A.; RODRIGUES, A.S.; FERRAZ, P.A.; RODRIGUES, A.S.; LOIOLA, M.V.G.; CHALHOUB, M. RIBEIRO FILHO, A. de L. Eficiência do Cipionato de Estradiol e do Benzoato de Estradiol em Protocolos de Indução da Ovulação Sobre a

Dinâmica Ovariana e Taxa de Concepção de Fêmeas Nerole Inseminadas em Diferentes Momentos. Archives of Veterinary Science, v.17, n.4, p.70-82, 2012.

AYRES, H.; PENTEADO, L.; TORRES JUNIOR, J.R.S.; SOUZA, J.R.; BARUSELLI, P.S. Taxa de Concepção de Vacas Nelore Lactantes Sincronizadas com Implante Auricular de Progestágeno Associado ao Benzoato ou ao Cipionato.

Acta Scienciae Veterinariae, v. 34, p.410, 2006.

BARUSELLI, P.S.; REIS, E.L.; MARQUES, M.O.; NASSER, LF.; BÓ, G.A. THE Use of Hormonal Treatments to Improve Reproductive Performance of Anestrous Beef Cattle in Tropical Climates. Animal Reproduction Science, v.82-83, p.479-486, 2004.

BARUSELLI, P.S.; AYRES, H.; SOUZA, A.H.; MARTINS, C.M.; GIMENES, L.U.; TORRES-JÚNIOR, J.R.S. Impactoda IATF na Eficiência Reprodutiva em Bovinos de Corte. In: SIMPÓSIO INTERNACIONAL DE REPRODUÇÃO ANIMAL APLICADA, 2, 2006, Londrina. Anais... Londrina, 2006. p.103-136.
BÓ, G.A.; ADAMS, G.O.; PIERSON, R.A.; MAPLETOFT, R.J. ${ }^{1}$ Exogenous Control of Folicular Wave Emergence in Cattle. Theriogenology, v.43, n.1, p.3134. 1995.

BÓ, G.A.; BARUSELLI, P.S.; MATINEZ, M.F. Pattern and Manipulation of Follicular Development in Bos indicus Cattle. Animal

Reproduction Science, v.78, p.307-326, 2003.

CARDOSO, B.L.; PESCARA, J.B.; VASCONCELOS, J.L.M. Protocolos de Inseminação Artificial em Tempo Fixo Para Vacas Mestiças Leiteiras. 2010. Disponível em:

http://www.abspecplan.com.br/upload/lib rary/iatf_vacas_mesticas.pdf. Acesso em: 17 set. 2015.

CREPALDI, G.A. Eficácia de Diferentes Protocolos de Indução da Ovulação e de Intervalos de Inseminação em Vacas de Corte Submetidas à IATF. 2009. 88f.

Dissertação (Mestrado em Medicina Veterinária) - Universidade de São Paulo, São Paulo.

DANIELI, T.; GRADELA, A CARNEIRO, T.; TORRES, D.V.; GROSSI, S. Aumento da eficiência reprodutiva de novilhas e vacas leiteira cíclicas com o uso de cipionato de estradiol no momento da retirada do CIDR. In: CONBRAVET. Anais... 2008. Disponível em:

$\leq$ http://www.sovergs.com.br/conbravet2 008/anais/cd/resumos/r0751-1.pdf,>. Acesso em: 04 mar. 2015.

FREITAS, B.G.; SALA, R.V.; VIEIRA, L.M.; MACEDO, G.G.; SANTOS de MIRANDA, M.; BARUSELLI, P.S. Resposta ovariana após o uso de Benzoato de Estradiol e Cipionato de Estradiol como indutores de ovulação em protocolos de sincronização para IATF 
Rev. Bras. Saúde Prod. Anim., Salvador, v.16, n.4, p.958-965out../dez.., 2015 http://www.rbspa.ufba.br ISSN 15199940

em fềmeas da raça holandesa. In:

REUNIÃO ANUAL DA SOCIEDADE BRASILEIRA DE TECNOLOGIA DE EMBRIÕES, 17, 2013, Praia do Forte, BA. Anais... Praia do Forte, BA, 2013.

INFORZATTO, G.R.; SANTOS, W.R.M.; CLIMENI, B.S.O.; DELLALIBERA, F.L.; FILADELPHO, A.L. Emprego de IATF (Inseminação Artificial em Tempo Fixo) Como Alternativa na Reprodução da Pecuária de Corte. Revista Cientifica de Medicina Veterinaria, v.6, n.11, 2008.

KOZICKI, L.E.; SEGUI, M.S.; FANTINI FILHO, J.C.; PRADO, F.R.A.; MATTE, F.; GLASER JUNIOR, P.; WEISS, R.R. A somatotrofina bovina (bST) e sua relação com o recrutamento folicular ovariano durante o ciclo estral de vacas. Archives of Veterinary Science, v.10, n. 1, p.35-44, 2005.

LUCY, M.C. Desafios na reprodução de vacas leiteiras de alta produção. In: CURSO NOVOS ENFOQUES NA PRODUÇÃO E REPRODUÇÃO DE BOVINOS, 15, 2011, Uberlândia. Anais.... Uberlândia, 2011. p. 91-102.

PFEIFER, L.F.M.; CORRÊA, M.N.; SCHMMIT, E.; VIEIRA, M.B.; MADRUGA, E.Á.; RABASSA, V.R. Uso de PGF2 $\alpha$ associado ao benzoato de estradiol para Inseminação artificial em tempo-fixo em vacas leiteiras.

Revista Brasileira de Agropecuária, v.11, n.3, p.347-350, 2005.

PINHEIRO, V.G.; SOUZA, A,F.; PEGORER, M.F.; SATRAPA, R.A.; ERENO, R.L.; TRINCA, L.A.;

BARROS, C.M. Effects of temporary calf removal and $\mathrm{eCG}$ on pregnancy rates to timed-insemination in progesteronetreated postpartum Nellore cows. Theriogenology. v.71, p.519-521, 2009.
RODRIGUES, C.A.; TEIXEIRA, A.A.; SOUZA A.H.; FERREIRA, R.M.; AYRES, H., BARUSELLI, P.S. Fatores que influenciam o sucesso de programas de IATF em gado de leite. In: SIMPÓSIO INTERNACIONAL DE REPRODUÇÃO ANIMAL APLICADA: BIOTECNOLOGIA DA REPRODUÇÃO EM BOVINOS, 2008. Londrina. Anais... Londrina, 2008. p.7894.

RODRIGUES, C.A; TEIXEIRA, A.H.; SOUZA,R.M.; Ferreira, R.M.; Ayres, H.; Baruselli, P.S. Fatores que Influenciam o Sucesso de Programas de IATF em Gado de Leite. In: SIMPÓSIO INTERNACIONAL DE REPRODUÇÃO ANIMAL APLICADA: BIOTECNOLOGIA DA REPRODUÇÃO EM BOVINOS, 3 , 2011, São Paulo. Anais... São Paulo: Universidade de São Paulo, 2011.

SALES, J.N.S.; CARVALHO, J.B.P.; CREPADI, G.A.; CIPRIANO, R.S.; JACOMINIA, J.O.; MAIO, J.R.G.; SOUZA, J.C.; NOGUEIRA, G.P. ; BARUSELLI, P. S. Effects of Two Estradiol Esters (Benzoate and Cypionate) on the Induction of Synchronized Ovulations in BosIndicus Cows Submitted to a Time Artificial Insemination Protocol. Theriogenology, v.78, p.510-516, 2012.

SARTORI, R.; FRICKE, P.M.; FERREIRA, J.C.P.; GINTHER, O.J.; WILTBANK, M. C. Follicular deviation and acquisition of ovulatory capacity in bovine follicles. Biology of

Reproduction, v.65, p.1403-1409, 2001.

Data de recebimento: 05/03/2015

Data de aprovação: 09/10/2015 